\title{
Blood Flow Restriction Effects on Amateur Soccer Player: More than Just Strength and Mass Gains?
}

\section{Besozzi Lorenzo*}

Department of Physiotherapy, Ola Grimsby Insitute/Rehability Lugano, Lugano, Swiss, Switzerland

*Corresponding Author: Besozzi Lorenzo, Department of Physiotherapy, Ola Grimsby Insitute/Rehability Lugano, Lugano, Swiss, Switzerland.
Received: May 10, 2021

Published: May 24, 2021

(C) All rights are reserved by Besozzi Lorenzo.

\begin{abstract}
Objective: To investigate effects of blood flow restriction (BFR) in pain modulation beyond other well-established effects on muscle gains after anterior cruciate ligament (ACL) surgery in a soccer player.

Design: Case study examining BFR training in a clinical rehabilitation setting.

Methods: BFR was utilized in a strength training protocol of the lower limbs after ACL surgery. Pain values on a visual analogue scale (VAS) were collected before and after the strength protocol's execution. The cross-sectional area of the thigh and isometric mean and peak force output during a squat were measured before the protocol execution.

Results: Minimal clinical important difference (MCID) of $20 \mathrm{~mm}$ on a 1 - $100 \mathrm{~mm}$ VAS was reported in both pre- and post-training values between the first data collection (T0) and the last one (T3). No improvements were reported in CSA values on the injured limb between T0-T3. Inconsistent values were reported in the isometric squat test: an increase of both mean and peak from T0 were reported in $\mathrm{T} 1$ and $\mathrm{T} 2$. Both values then decreased again in $\mathrm{T} 3$, below $\mathrm{T} 0$ values.
\end{abstract}

Conclusion: BFR may play an essential role in pain modulation after ACL surgery.

Keywords: ACL; Pain; Rehabilitation; Soccer

\section{Introduction}

Anterior cruciate ligament (ACL) injuries are common in sports, especially in those that apply severe stress to the knee joint, such as soccer, where a change of direction, jumping, landings and high speed running occur with and without contact [1]. The impact of these injuries on athletes is significant, considering it takes between 8 - 12 months before the potential to return to play (RTP) [2]. Furthermore, only $65 \%$ of the players who suffer an ACL rupture can return to their previous level of performance after 3 years from injury [3] and that different clinical consequences, such as arthritis, are often observed [4]. Specifically in football, or soccer, studies by the Union of European Football Association (UEFA) showed an increase number of ACL injuries on matchdays vs. practice which may be due to higher physical demand of competition [5]. The player's position can play a significant role in developing such an injury. The two positions at highest risk of suffering an ACL injury are the defender (43\% incidence) and the midfielder (31\% incidence) [6].

Non-contact injuries are the most common injuries found in football: pressing the opponent being the first scenario for the 
number of cases reported, followed by regaining balance and landing after heading the ball, while contact injuries in football occur typically due to tackle situations, which create a valgus momentum of the knee, leading to ACL rupture [7].

Long-term symptoms at the knee joint are may be present after an ACL tear [8]. Pain is one of the most frequently reported symptoms after surgery [9]. During the rehabilitation process, pain is often still present and can significantly delay the achievements needed in the rehab's first phases. There is a lack of literature about pain management after surgery through exercise, however several interventions have been described including transcutaneous electrical nerve stimulation (TENS), cryotherapy and systemic pharmacologic therapies [10].

According to the American College of Sports Medicine, muscular strength and hypertrophy can be achieved through exercising 2 - 3 times a week with intensities higher than $65 \%$ of one's individual one-repetition maximum (1RM) [11]. It may be challenging to reach these training intensities when people are recovering from surgery due to pain and inability to tolerate loads, which can be a limiting factor in the strength and hypertrophy gains, especially during a long-term rehabilitation process. ACL tears in people who underwent surgery tend to demonstrate muscle weakness, especially in the quadriceps [12,13], reduced quality of life [14] and lower RTP rate [15]. To avoid such conditions and create better outcomes from rehabilitation, Blood Flow Restriction (BFR) training can be implemented in the early stages, right after surgery [16].

BFR training consists of using a tourniquet on a proximal limb during low-intensity exercise to reduce $80 \%$ of arterial blood flow while blocking the venous outflow completely. The block that the tourniquet creates on the limb stimulates the creation of an anaerobic system, similar to the one needed to perform training at high intensities over $65 \% 1 \mathrm{RM}$, despite working with lower mechanical loads [17]. Therefore, BFR can lead to similar results as traditional high mechanical training through physiological effects.

Lactate production created during the use of BFR leads to the recruitment of larger motor units, the ones that form fast-twitch muscles, which will then be activated to avoid failure even if the subject is working with low resistances (20 - 30\% 1RM) [18]. Lactate production will also stimulate the pituitary gland to release growth hormone $(\mathrm{GH})$, which is linked to higher collagen produc- tion. Meanwhile, the lower resistances used during the exercise will not result in collagen breakdown, leading to a positive collagen turnover [19]. Insulin growth factor 1 (IGF 1) is a protein linked to muscle growth that activates satellite cells, the precursors of myocytes, and fuses them into the muscle fibers [20] which is activated by $\mathrm{GH}$. BFR, GH, and lactate production also activate the mammalian target of rapamycin complex 1 (MTORC1), a complex responsible for modulating the muscle growth process [21].

Although the effects of BFR on muscular architecture in terms of trophism, has been well established, its effect on pain has barely been investigated. The British National Health Service (NHS) investigated outcomes of BFR application following ACL surgery. Subjects experienced a decrease in pain after a 9-week protocol [22], however, within each session, pre/post pain levels (before-after BFR protocol) were not assessed. The purpose of this case study is to evaluate the immediate impact of BFR in reducing one individual's pain during a strengthening protocol. A secondary purpose is to assess the impact of BFR on strength and mass gains for the same subject.

\section{Materials and Methods}

This study will analyze thigh cross-sectional area (CSA), visual analog scale (VAS) and strength output through an encoder, once a week, over four weeks. CSA values will be measured manually in centimeter before the warm-up to verify any previous evaluation changes. Pain values will be evaluated through the VAS scale before the warm-up and 5 minutes after the end of the BFR protocol. Through the use of the encoder, a mean and a peak force will be evaluated during an isometric squat strength test, and this test will also be performed after the warm-up. The subject who participated in the study provided signed informed consent in compliance with the declaration of Helsinki [23].

\section{Blood flow restriction (BFR) unit}

In this study, the DELFIC PTS BFR device was used. This device belongs to the third generation of tourniquets which represents the safest for clinical use [24] this is because these devices show a series of features, like self-calibration to establish correct pressure, ability to set limits for pressure, and inflation time and automatic measurement of limb occlusion pressure (LOP) which represents the lowest pressure to reach the target occlusion on a given patient. Cuffs are then placed at the proximal limb to be trained and linked through a tube to the device. DELFIC PTS device comes with 
three different "Variable Countour" cuffs that can be adjusted to the shape of the limb to enhance comfort, reduce mechanical shearing, and require lower pressures to occlude the flow [25].

The therapist starts the LOP measurement by having the patient lying as motionless as possible in the supine position. This test calculates the personalized pressure to be used during training, which will be $80 \%$ of calculated pressure when training on lower limbs and $50 \%$ when training upper limbs. The subject selected for this study performed BFR standard protocol of 30-15-15-15 repetitions divided into four sets performing a squat with the help of TRX(C) with 30-second rest between every set, in addition to a strengthening protocol.

\section{Encoder device}

Measurements of strength output during an isometric squat with knees flexed at $90^{\circ}$ were recorded with "Desmotec D.plus pininfarina isoinertial device" (Figure 1). This device has integrated load cells on the ground platform. The Isometric Test consisted of expressing the maximum force from a squat position where both knees and hips were bent at $90^{\circ}$. The patient had to wear a vest that was connected through an adjustable rope to the platform. The therapist adjusted the length of the rope in order to place the person to be tested in the proper starting position. During the test, the patient was asked to push as hard as he could for the full test duration of 15 seconds, as if he was trying to get back to a standing position. The load cell "D.load cells" registered the force applied on them in terms of $\mathrm{KG} / \mathrm{cm}^{2}$, showing a mean value and the max peak force for the 15 second test. "Desmotec D.plus pininfarina" is an isoinertial workstation, with a rotatory encoder installed to perform a different test to evaluate strength, power, and balance outputs in several tasks, like squatting and lunges. Test-retest reliability and validity of a flywheel squat test using the ergomemeter (D11 Full, Desmotec Biella) demonstrated excellent relative reliability with an intraclass correlation coefficient (ICC) score of $>0.9$ and an acceptable coefficient of variation (CV) of $<5 \%$ between different days in strength outputs [26].

\section{Visual analogue scale (VAS)}

A visual analouge scale (VAS) to assess pain was used before warm-up and after completing the protocol to evaluated if the BFR intervention modifies pain. VAS is formed by a line with numbers written on it ranging from 0 - 100 millimeters, where 0 stands for

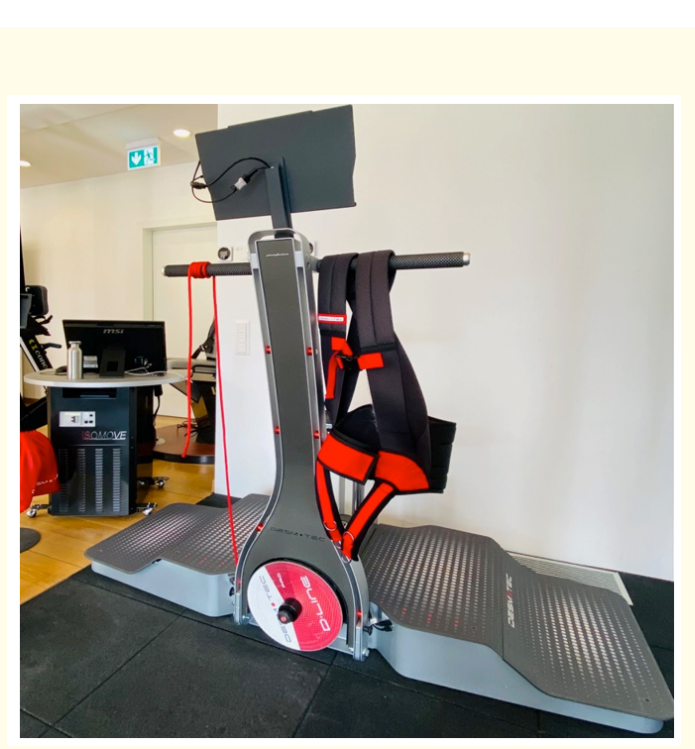

Figure 1: Desmotec D.Plus Pininfarina device.

"No pain sensation" and 100 represented by "Maximum pain you felt since you injured your knee." The VAS scale has been widely used between practitioners, and it has been clinically validated [27].

To set test-retest reliability and evaluate the intervention's efficacy, an MCID (minimal clinically important improvement) was established. RCTs studies indicate MCID for VAS to be between 11.1 to $19.9 \mathrm{~mm}$ [28] without referring to a specific part of the body while $19.9 \mathrm{~mm}$ was the MCID indicated for the knee [29]. In this study MCID is defined as $16.9 \mathrm{~mm}$, which is a mean of the 3 values previously mentioned, avaiable in the literature.

\section{Cross-sectional area (CSA)}

A cross-sectional area (CSA) is a cross-section of a muscle, perpendicular to its fibers, generally at the largest point. This study analyzed if, through the use of BFR, an increase in CSA could have been reached. There is a contrast to whether a relationship between muscle CSA and force production exists in literature [30]. CSA is usually measured using an MRI scan [31]. Unfortunately, the use of MRI technology is not possible for this case study due to cost and difficult access to repeated MRI scans, especially during the COVID-19 pandemic. The CSA measurements were taken manually 
in centimeters $(\mathrm{cm})$ at the distance of $6 \mathrm{~cm}$ and $12 \mathrm{~cm}$ from the patella's superior board, which took place before the training session. There are flawed findings on MCID relationship to CSA, so a distribution-based method [32] will be used to determine whether or not the intervention produced meaningful changes. Based on this method, a standardized change of $0.8 \mathrm{~cm}$ on the CSA will be considered as MCID.

\section{Case Presentation}

The subject was a 22 years old male non-professional soccer player. He tore his left knee ACL while performing a sudden change of direction to the right with the left foot planted on the ground during pre-season training (i.e., external rotation of the tibia and the femur's internal rotation creating a valgus momentum). The Injury affected only the ACL as the MRI scan did not find collateral ligament or meniscal tears. The subject did not have a history of previous knee injury and underwent surgery three weeks later. The ACL repair was performed through the use of a Semitendineous graft, and no adverse events were reported during the procedure. The protocol started four weeks after the surgery. During the three weeks prior to starting the protocol, the subject received physiotherapy to restore complete extension of the knee, allowing painfree range of motion, and cryotherapy was initiated to prepare the athlete for the strengthening program.

The strengthening protocol (Table 1) was performed twice a week for four weeks with a day of rest between sessions. Data acquisition took place during the first session of the week for a total of four data collection periods (T0, T1, T2, T3). CSA measurements and VAS values were recorded before starting the stationary bike warm up and 5 minutes after the last excercise of the session; the $90^{\circ}$ isometric squat test on the encoder was only collected after warm up.

\begin{tabular}{|l|c|}
\hline \multicolumn{1}{|c|}{ Excercise } & Sets/Repetitions \\
\hline Stationary bike & 8 Minutes \\
\hline Leg press (30\% $1 \mathrm{Rm})$ & 4 sets/8 repetitions \\
\hline $\begin{array}{l}\text { Leg curl isometric holds at } 45^{\circ} \text { knee } \\
\text { flexion }\end{array}$ & 4 sets $/ 8$ repetitions \\
\hline Sitting monolateral heel raise & 4 sets $/ 8$ repetitions \\
\hline $\begin{array}{l}\text { BFR squat to } 90^{\circ} \text { knee flexion TRX } \\
\text { (suspension system) }\end{array}$ & \\
\hline
\end{tabular}

Table 1: Strengthening protocol performed during the study.

\section{Results}

CSA

Regarding cross sectional area, the patient presented linear values through the process, with no change in the measurements between the first evaluation (T0) and the last evaluation (T3), where a CSA value of $43.5 \mathrm{~cm}$ was reported in both times on the injured leg (left). On the non-involved right leg, where BFR was not applied, an increase of $1.5 \mathrm{~cm}$ was noted from the first evaluation (T0) and the last evaluation at the end of the study (T3), as notable in figure 2.

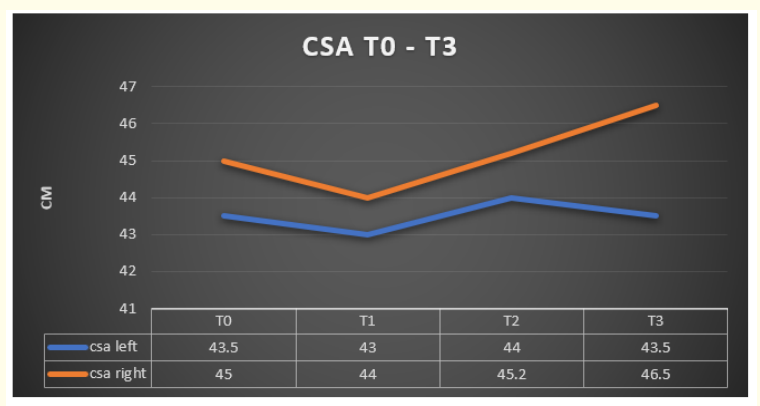

Figure 2: CSA left= Cross-Sectional Area left leg, CSA right= Cross-Sectional Area right leg, $\mathrm{T} 0=$ first data acquisition, $\mathrm{T} 1=$ second data acquisition, T2= third data acquisition, $\mathrm{T} 3=$ fourth and last data acquisition.

\section{Isometric squat strength test- Encoder}

The test records the mean value of force expressed in Kilograms (Kg) during the whole test and the maximum force peak reached in the test. An increase of force production while the program was carried on was showed in mean strength outputs between (T0) $416.2 \mathrm{Kg}$ and (T2) $446 \mathrm{Kg}$ while a decrease in the values is shown in the last test performed (T3) $407.9 \mathrm{Kg}$. The peak force reached was inconsistent between the sessions, with the highest peak of force produced being recorded in the second session (T1), as notable in figure 3.

Balance asymmetries in strength outputs between the two legs are also reported from the encoder while performing the test. This variable measures in percentage (\%) which of the two limbs is producing more force. As shown, in figure 4, the patient was able to improve the ratio of force production from the left (injured) leg to 


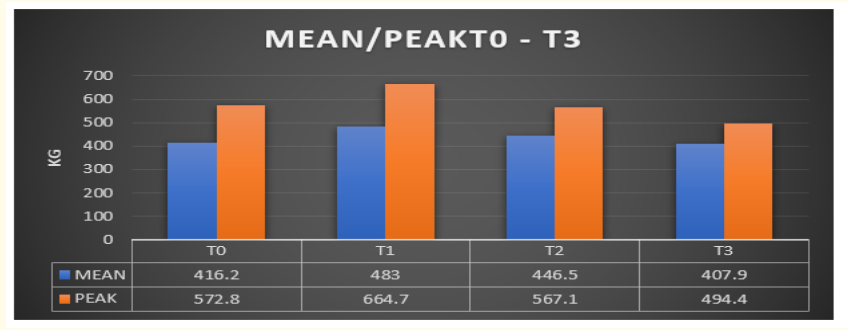

Figure 3: Compares mean force production, the peak reached, and the trend of these values during the study. Mean= mean of force values produced during the whole duration of the test, Peak= peak of maximum force output registered during the test, $\mathrm{T} 0=$ first data acquisition, $\mathrm{T} 1=$ second data acquisition, $\mathrm{T} 2=$ third data acquisition, $\mathrm{T} 3=$ fourth and last data acquisition.

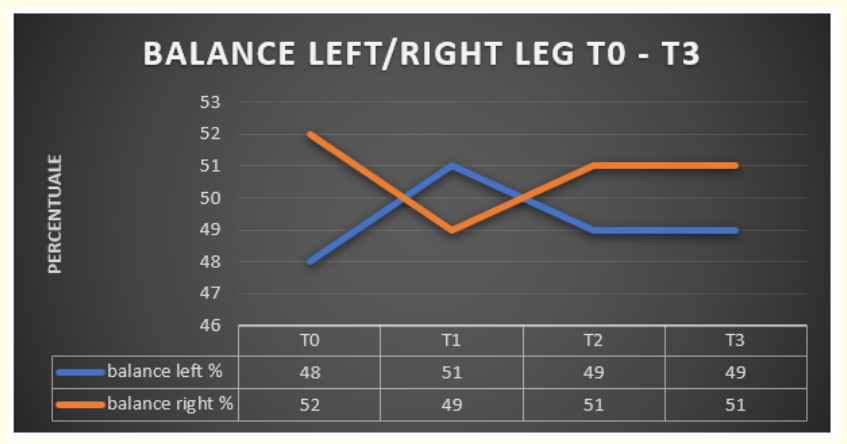

Figure 4: Balance between left and right leg in generating force during the $90^{\circ}$ isometric squat strength test. Balance left $\%=$ percentage of force output generated by the left limb, balance right $\%=$ percentage of force output generated by the right leg. $\mathrm{T} 0=$ first data acquisition, $\mathrm{T} 1=$ second data acquisition, $\mathrm{T} 2=$ third data acquisition, T3= fourth and last data acquisition.

$49 \%$ (T3) from the starting value of $48 \%$ recorded at the first test (T0).

Visual analogue scale (VAS)

Patient reported pain VAS scores between the first evaluation (T0) and the last treatment (T3) resulted in a $20 \mathrm{~mm}$ decrease. As reported in figure 5 if we consider the values after using BFR, pain sensation is reduced up to $50 \%$ from the first training session (T0) and the last evaluation (T3). It is of relevance to underline that the pain senstaion recorded through the VAS scale has been constantly decreasing from the second evaluation (T1) to the last one (T3) has shown in figure 5 .

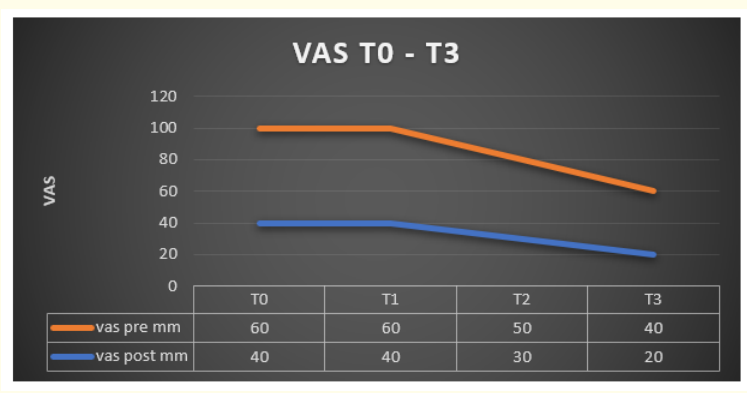

Figure 5: VAS trend during the study. VAS post= pain sensation score given by the patient after the intervention, VAS pre= pain sensation score given by the patient before the intervention, $\mathrm{T} 0=$ first data acquisition, $\mathrm{T} 1=$ second data acquisition, $\mathrm{T} 2=$ third data acquisition, T3= fourth and last data acquisition.

\section{Discussion}

The CSA outcomes showed no difference from T0 to T3 on the injured limb (left); the MCID threshold $(0.8 \mathrm{~cm})$ was not met. Thus, unlike a previous study [33] there was no increase in muscle hypertrophy following BFR application.

The encoder $90^{\circ}$ isometric squat strength test data was not consistent with expected outcomes. An increase of both mean power and peak output was recorded at T1 when compared to T0. However, T2 showed a decrease in both mean and peak values when compared to T1, but still an increase when compared to T0. Furthermore, T3 showed a decrease of both mean and peak values to the lowest levels measured during the intervention.

This could be biased from several factors (tiredness, sleep routine, work routine) on the day of the test. If a comparison with the literature is made, other studies shown an increase of strength output evaluated through 10RM strength on a leg press over a study period of 8 weeks [34]. In this case study, data evaluation occured 
over a period which was $50 \%$ shorter ( 4 weeks vs 8 weeks). Furthemore, measurements were taken in a standing position by an encoder, and this may represent a more functional position relating to a sport, soccer, which is played in a standing position rather than an non-functional position like a sitting leg press.

Lower limb symmetry was assessed using the $90^{\circ}$ isometric squat strength test. Through this parameter, it is possible to see how the involved left leg gained strength during the intervention resulting in improved limb symmetry, reaching a 49\% at T3 while at T0 the balance reported was $48 \%$. The aspect of force output symmetry using the standing encoder with the same test position has not been investigated in previous studies.

BFR intervention for pain modulation has not been fully elicidated in the literature, especially the influence of BFR has on pain values within the same sessions. The study conducted by the NHS [35] shows a decrease in pain values after 8 weeks of intervention, which could also be linked to normal physiological reduction in pain after the inflammatory phase or due to a significant passage of time ( 8 weeks). In this case study, every time the subject was asked to report his pain sensation on the VAS scale, the values were lower after the intervention than the one he reported before the protocol was performed. When considering the VAS data, the MCID threshold of a $20 \mathrm{~mm}$ decrease was met in each session and at the end of the study (T3). This could be due to effects of exercise on cortical activation/inhibition pathways [36] however, the pain pathways through which BFR may influences pain sensations still needs to be investigated.

\section{Limitations of the Study}

The subject's working routine and the uncontrolled aspect of the subject's regular life such as sleeping, emotional side, and diet were not controlled. They could have played a significant role in the outcomes evaluated, especially in the isometric squat strength test. Another limit present in the study was the lack of access to more accurate evaluation instruments like MRI, which is considered the goal standard when evaluating CSA.

\section{Conclusion}

BFR is an emerging technology in the rehabilitation setting. Its efficacy for strength gains and muscle mass are well established [37]; however, there is a gap in the literature in regarding any immediate effect BFR might have on pain modulation, especially after
ACL reconstruction. To the author's knowledge, prior BFR studies have not investigated or reported immediate within-session pain modulation following BFR treatment. This case study indicates BFR may decrease pain sensation after application duirng early management of post surgical ACL, where pain is a limiting factor for progress during rehabilitation. A randomized clinical trial is suggested in order to verify this outcome on a wider population and to study if there may be connections between the increase in strength values and reduced perceived pain.

\section{Bibliography}

1. Gornitzky AL., et al. "Sport-Specific Yearly Risk and Incidence of Anterior Cruciate Ligament Tears in High School Athletes: A Systematic Review and Meta-analysis". American Journal of Sports Medicine 44.10 (2016): 2716-2723.

2. Zaffagnini S., et al. "Return to sport after ACL reconstruction: how, when and why? A narrative review of current evidence". Joints 3.1 (2015): 25-30.

3. Waldén M., et al. "ACL injuries in men's professional football: a 15-year prospective study on time trends and return-to-play rates reveals only $65 \%$ of players still play at the top level 3 years after ACL rupture". British Journal of Sports Medicine 50.12 (2016): 744-750.

4. Von Porat A., et al. "High prevalence of osteoarthritis 14 years after an anterior cruciate ligament tear in male soccer players: a study of radiographic and patient relevant outcomes". Annals of the Rheumatic Diseases 63.3 (2004): 269-273.

5. Waldén M., et al. “ACL injuries in men's professional football: a 15-year prospective study on time trends and return-to-play rates reveals only $65 \%$ of players still play at the top level 3 years after ACL rupture". British Journal of Sports Medicine 50.12 (2016): 744-750.

6. Della Villa F., et al. "Systematic video analysis of ACL injuries in professional male football (soccer): injury mechanisms, situational patterns and biomechanics study on 134 consecutive cases". British Journal of Sports Medicine 54.23 (2020): 14231432.

7. Waldén M., et al. "The epidemiology of anterior cruciate ligament injury in football (soccer): a review of the literature from 
a gender-related perspective". Knee Surgery, Sports Traumatology, Arthroscopy 19.1 (2011): 3-10.

8. Lohmander LS., et al. "High prevalence of knee osteoarthritis, pain, and functional limitations in female soccer players twelve years after anterior cruciate ligament injury". Arthritis and Rheumatology 50.10 (2004): 3145-3152.

9. Elaqoul A., et al. "Postoperative Pain Among Patients After Day-Case Surgery". Plastic Surgical Nursing 37.4 (2017): 130136.

10. Chou R., et al. "Management of Postoperative Pain: A Clinical Practice Guideline From the American Pain Society, the American Society of Regional Anesthesia and Pain Medicine, and the American Society of Anesthesiologists' Committee on Regional Anesthesia, Executive Committee, and Administrative Council [published correction appears in". Journal Pain 17.4 (2016): 508-510.

11. Donnelly Joseph E., et al. "American College of Sports Medicine Position Stand. Appropriate physical activity intervention strategies for weight loss and prevention of weight regain for adults". Medicine and Science in Sports and Exercise 41.2 (2009): 459-471.

12. Palmieri-Smith Riann M., et al. "Maximizing quadriceps strength after ACL reconstruction". Clinics in Sports Medicine 27.3 (2008): 405-424.

13. Snyder-Mackler Lynn., et al. "Strength of the quadriceps femoris muscle and functional recovery after reconstruction of the anterior cruciate ligament. A prospective, randomized clinical trial of electrical stimulation". Journal of Bone and Joint Surgery 77.8 (1995): 1166-1173.

14. Lepley AS., et al. "Quadriceps neural alterations in anterior cruciate ligament reconstructed patients: A 6-month longitudinal investigation". Scandinavian Journal of Medicine and Science in Sports 25.6 (2015): 828-839.

15. Ardern Clare L., et al. "Fifty-five per cent return to competitive sport following anterior cruciate ligament reconstruction surgery: an updated systematic review and meta-analysis including aspects of physical functioning and contextual factors". British Journal of Sports Medicine 48.21 (2014): 1543-1552.
16. Day B. "Personalized Blood Flow Restriction Therapy: How, When and Where Can It Accelerate Rehabilitation After Surgery?" Arthroscopy 34.8 (2018): 2511-2513.

17. Meyer RA. "Does blood flow restriction enhance hypertrophic signaling in skeletal muscle?" Journal of Applied Physiology 100.5 (1985): 1443-1444.

18. Yasuda Tomohiro., et al. "Effects of low-intensity bench press training with restricted arm muscle blood flow on chest muscle hypertrophy: a pilot study". Clinical Physiology and Functional Imaging 30.5 (2010): 338-343.

19. Schoenfeld BJ. "Potential mechanisms for a role of metabolic stress in hypertrophic adaptations to resistance training". Sports Medicine 43.3 (2013): 179-194.

20. Owens JG and Hsu J. "Dedicated professionals givre the injured another chance to serve". Journal of bone and joint surgeryJournal of orthopedic sports physical therapy special report: It takes a team (2013).

21. Dreyer Hans C., et al. "Leucine-enriched essential amino acid and carbohydrate ingestion following resistance exercise enhances mTOR signaling and protein synthesis in human muscle". American Journal of Physiology-Endocrinology And Metabolism 294.2 (2008): E392-E400.

22. Hughes L., et al. "Comparing the Effectiveness of Blood Flow Restriction and Traditional Heavy Load Resistance Training in the Post-Surgery Rehabilitation of Anterior Cruciate Ligament Reconstruction Patients: A UK National Health Service Randomised Controlled Trial". Sports Medicine 49.11 (2019): 1787-1805.

23. World Medical Association. World Medical Association Declaration of Helsinki: ethical principles for medical research involving human subjects". The Journal of the American Medical Association 310.20 (2013): 2191-2194.

24. McEwan JA. "Tourniquet use and care (2014).

25. AORN. Recommended practices for use of the pneumatic tourniquet in the preoparative practice setting. In: preoparative standards and recommended practices for inpatient and ambulatory settings. 2014 ed. Denver, CO AORN Inc (2014): 183-208. 
26. Marco Beato., et al. "Validity and reliability of a flywheel squat test in sport (2020).

27. Karcioglu Ozgur., et al. "A systematic review of the pain scales in adults: which to use?" The American Journal of Emergency Medicine 36.4 (2018): 707-714.

28. Tubach Florence., et al. "Evaluation of clinically relevant changes in patient reported outcomes in knee and hip osteoarthritis: the minimal clinically important improvement". Annals of the Rheumatic Diseases 64.1 (2005): 29-33.

29. Ehrich Elliot W., et al. "Minimal perceptible clinical improvement with the Western Ontario and McMaster Universities osteoarthritis index questionnaire and global assessments in patients with osteoarthritis". The Journal of Rheumatology 27.11 (2000): 2635-2641.

30. Jones Eric J., et al. "Cross-sectional area and muscular strength". Sports Medicine 38.12 (2008): 987-994.

31. Barber-Westin., et al. "Blood Flow-Restricted Training for Lower Extremity Muscle Weakness due to Knee Pathology: A Systematic Review". Sports Health 11.1 (2019): 69-83.

32. Mouelhi Yosra., et al. "How is the minimal clinically important difference established in health-related quality of life instruments? Review of anchors and methods". Health and Quality of Life Outcomes 18 (2020): 1-17.

33. Hughes L., et al. "Examination of the comfort and pain experienced with blood flow restriction training during post-surgery rehabilitation of anterior cruciate ligament reconstruction patients: A UK National Health Service trial". Physical Therapy in Sport 39 (2019): 90-98.

34. Hughes L., et al. "Examination of the comfort and pain experienced with blood flow restriction training during post-surgery rehabilitation of anterior cruciate ligament reconstruction patients: A UK National Health Service trial". Physical Therapy in Sport 39 (2019): 90-98.

35. Hughes L., et al. "Examination of the comfort and pain experienced with blood flow restriction training during post-surgery rehabilitation of anterior cruciate ligament reconstruction patients: A UK National Health Service trial". Physical Therapy in Sport 39 (2019): 90-98.
36. Lowe CJ., et al. "Effects of Moderate Exercise on Cortical Resilience: A Transcranial Magnetic Stimulation Study Targeting the Dorsolateral Prefrontal Cortex". Psychosomatic Medicine 79.2 (2017): 143-152.

37. Day B. "Personalized Blood Flow Restriction Therapy: How, When and Where Can It Accelerate Rehabilitation After Surgery?" Arthroscopy 34.8 (2018): 2511-2513.

Volume 4 Issue 6 June 2021

(C) All rights are reserved by Besozzi Lorenzo. 